\section{Acute kidney injury}

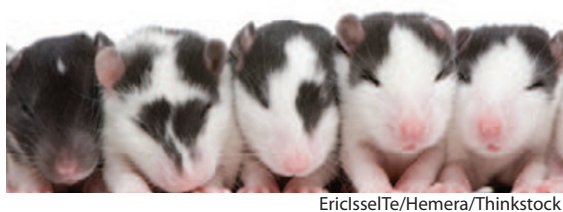

Stojanović and colleagues examined the sensitivity of KIM- 1 and cystatin-C, two biomarkers of renal injury, and investigated whether erythropoietin protects the kidneys of rat pups exposed to global ischemia in utero. As measured by both biomarkers, absolute injury scores were significantly lower in pups that received darbepoetin-a. See page 11

\section{Tobacco and adiposity}

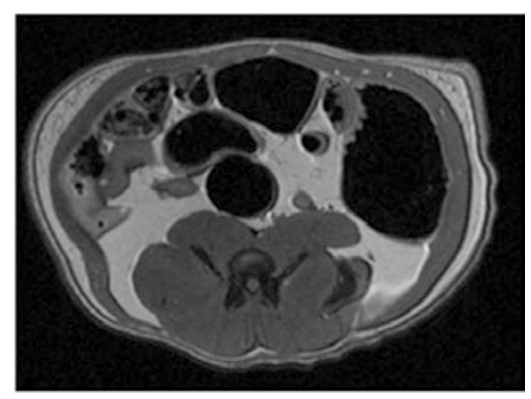

Zinkhan and coinvestigators

hypothesized that in utero exposure to tobacco smoke would increase visceral adiposity and alter the glucocorticoid pathway in young adult rats. Pregnant rats were exposed to cigarette smoke from E11.5 to term. Their newborns exhibited increased visceral adiposity and glucocorticoid pathway alterations in a sex-specific manner. See page 17

\section{Caffeine and developing white matter}

Caffeine is widely used to treat apnea of prematurity, but the standard dosing regimen is not always sufficient to prevent apnea. Atik et al. sought to determine the impact of daily

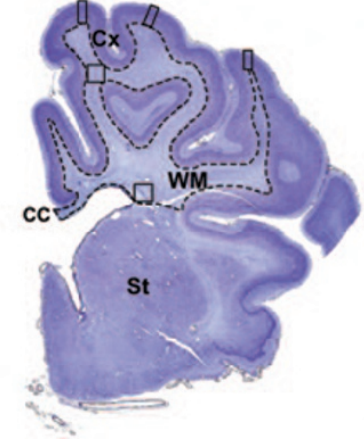

high-dose caffeine administration on developing ovine brain white matter. Caffeine or saline was administered to pregnant sheep. Necropsy showed no apparent structural alterations in white or gray matter resulting from daily high-dose caffeine. See page 54

\section{Oxygenation during feeding}

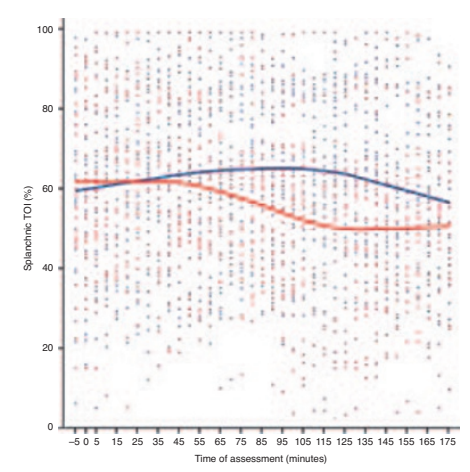

Corvaglia et al. investigated the differences between bolus and continuous feeding with respect to cerebral and splanchnic oxygenation in healthy preterm infants. They monitored oxygenation trends during feeding and found that only splanchnic oxygenation was influenced by the feeding method. See page 81

\section{Perinatal infection and encephalopathy}

Jenster and colleagues investigated whether maternal or neonatal infection is associated with adverse outcomes in term newborns with encephalopathy. Clinical records
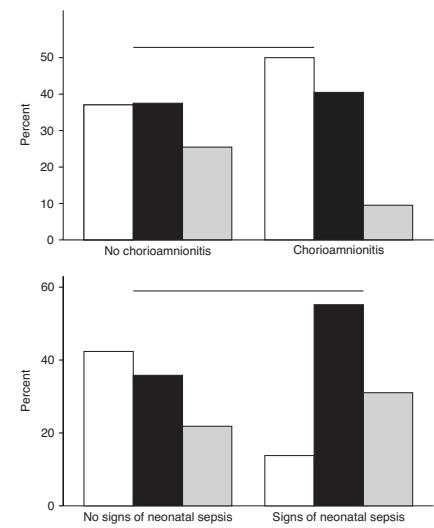

for 258 term newborns with encephalopathy were examined for signs of chorioamnionitis and sepsis. The results suggest that the timing of infection and inflammatory response may influence whether infection potentiates or mitigates injury in term newborns. See page 93

\section{Growth hormone and arm growth}

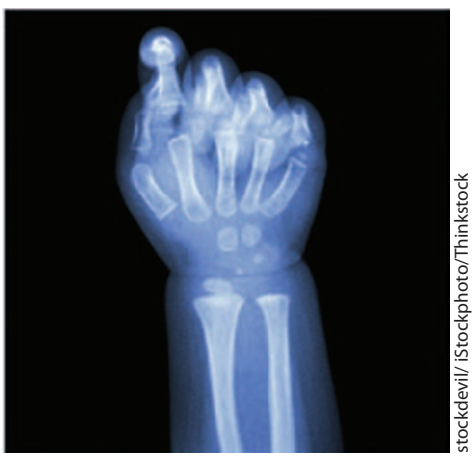

The process of growth and maturation of long and short bones of the hand differs from that for the carpal cuboid bones. Even and coauthors assessed the impact of growth hormone (GH) on the two processes of bone maturation. Both GH-deficient children and children with idiopathic short stature received $\mathrm{GH}$ treatment for two years. The dominant effect of $\mathrm{GH}$ appeared to be on growth of the carpal cuboid bones, with a milder effect on the long and short bones of the hand.

See page 109 